\title{
Design of Magnetic Flux Feedback Controller in Hybrid Suspension System
}

\author{
Wenqing Zhang, Jie Li, Kun Zhang, and Peng Cui \\ College of Mechatronics Engineering and Automation, National University of Defense Technology, Changsha, Hunan 410073, China \\ Correspondence should be addressed to Wenqing Zhang; zwq197566@163.com
}

Received 1 June 2013; Revised 1 September 2013; Accepted 15 September 2013

Academic Editor: Xinkai Chen

Copyright (c) 2013 Wenqing Zhang et al. This is an open access article distributed under the Creative Commons Attribution License, which permits unrestricted use, distribution, and reproduction in any medium, provided the original work is properly cited.

\begin{abstract}
Hybrid suspension system with permanent magnet and electromagnet consumes little power consumption and can realize larger suspension gap. But realizing stable suspension of hybrid magnet is a tricky problem in the suspension control sphere. Considering from this point, we take magnetic flux signal as a state variable and put this signal back to suspension control system. So we can get the hybrid suspension mathematical model based on magnetic flux signal feedback. By application of MIMO feedback linearization theory, we can further realize linearization of the hybrid suspension system. And then proportion, integral, differentiation, magnetic flux density B (PIDB) controller is designed. Some hybrid suspension experiments have been done on CMS04 magnetic suspension bogie of National University of Defense Technology (NUDT) in China. The experiments denote that the new hybrid suspension control algorithm based on magnetic flux signal feedback designed in this paper has more advantages than traditional positioncurrent double cascade control algorithm. Obviously, the robustness and stability of hybrid suspension system have been enhanced.
\end{abstract}

\section{Introduction}

A hybrid electromagnet made of permanent magnet and electromagnet consumes little power consumption and can realize larger suspension gap. So the hybrid suspension traffic will have more developments in the future. The CMS04 maglev vehicle (Figure 1) designed by National University of Defense Technology (NUDT) has been running above 20 thousand kilometers safely in the national mid-low speed maglev experiment field of Tangshan city in China. Hybrid suspension has high properties of suspension control system; therefore, promoting the robustness and stability of maglev control system is the destination of control engineers. Literature [1] founded the hybrid suspension model based on current feedback. Because the magnetic flux feedback control algorithm has wider control parameters zone and minor overshoot [2], this algorithm has been applied successfully in maglev vehicle of Britain airdrome line. Goodall gives emphasis to the advantages of the magnetic flux signal. Literature [3] mainly designs a robust fuzzy-neural-network control (RFNNC) scheme for the levitated positioning of the linear maglev rail system with nonnegative inputs.
A Maglev system is modeled by two self-organizing neuralfuzzy techniques to achieve linear and affine Takagi-Sugeno (T-S) fuzzy systems [4]. The influence of the PID control parameters on the response performance of the system is studied by using the MATLAB SISO [5]. Zhang et al. designed a detailed discourse upon the key technology involved in maglev system, and the method has been carried out in Shanghai maglev traffic on-site, and the results are very significant [6]. Wai and Lee $[7,8]$ has designed an adaptive fuzzy-neural-network control (AFNNC) scheme by imitating a sliding-mode control (SMC) strategy for a magneticlevitation (maglev) transportation system. Based on sliding mode control with the feedback linearization, a kind of nonlinear control strategy of electrical Maglev air gap was offered the design method of the system was researched [9]. A nonlinear robust control design for the levitation and propulsion of a magnetic levitation (maglev) system is presented, and a proposed recursive controller is designed using nonlinear state transformation and Lyapunov's direct method in order to guarantee global stability for the nonlinear maglev system [10]. To sum up, the control method based on magnetic flux signal feedback has a bright prospect. 


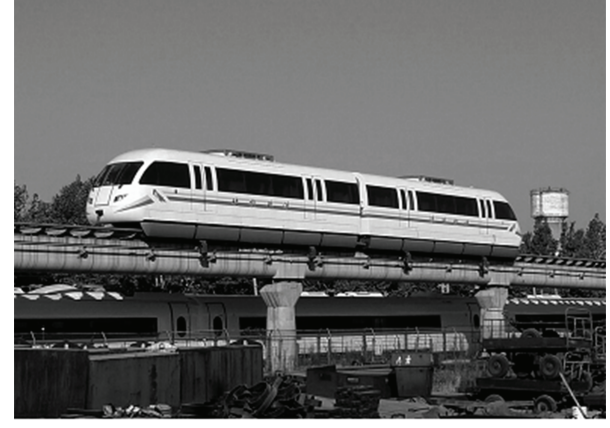

FIGURE 1: CMS04 maglev vehicle in National mid-low speed maglev experiment field of Tangshan city.

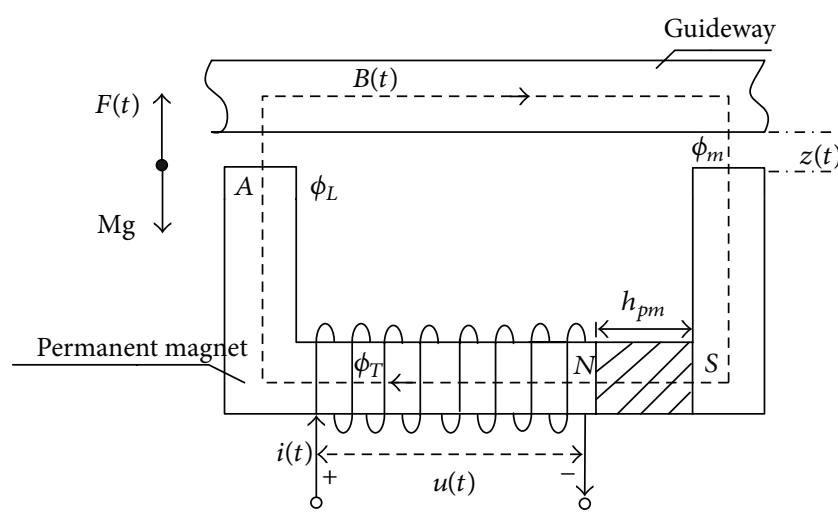

FIGURE 2: Simple model of hybrid suspension system.

\section{Modeling of Magnetic Flux Feedback Suspension System}

The hybrid suspension system model based on magnetic flux feedback is given in Figure 2.

Illuminating signs of Figure 2 are showing in Table 1.

Assume the following:

(1) magnetic flux formula $\phi_{T}=\phi_{L}+\phi_{m}$, neglect the leaking flux, namely, $\phi_{L}=0$,

(2) neglect the magnetic resistance of iron core and the track, assume the magnetic field potential distribute on the suspension gap evenly;

(3) suspension track has infinite rigidity with regard to the electromagnetic, so neglect deformation and the elastic vibration of track.

According to the magnetic ampere theorem, we get magnetic motive force

$$
F_{m}=\oint_{L} H \cdot d l=N i+H_{c} h_{p m} .
$$

Calculate the magnetic flux

$$
\Phi(z, i)=\frac{F_{m}}{R_{m}}=\frac{\mu_{0} A\left(N i+H_{c} h_{p m}\right)}{2 z(t)+h_{p m} / \mu_{r}} .
$$

TABLE 1: Symbols of hybrid suspension system.

\begin{tabular}{ll}
\hline Signs & Signification \\
\hline$\phi_{m}$ & Gap flux \\
$\phi_{T}$ & Main flux \\
$\phi_{L}$ & Leaking flux \\
$\psi$ & Gap flux linkage \\
$u(t)$ & Voltage of magnetic coil \\
$W_{m}$ & Magnetic field energy in bulk V \\
$R$ & Resistance of magnetic coil \\
$B$ & Gap flux density \\
$\mu_{0}$ & Magnetic permeability of atmosphere \\
$\mu_{p m}$ & Magnetic permeability of permanent magnet \\
$\mu_{r}$ & Magnetic permeability of permanent magnet \\
$H_{c}$ & Magnetic coercive force of permanent magnet \\
$F_{m}$ & Magnetic field potential \\
$R_{m}$ & Magnetic resistance \\
$A$ & Magnetic pole area \\
$i(t)$ & Current of magnetic coil \\
$w_{m}$ & Magnetic field energy density \\
$N$ & Turn of magnetic coil \\
$z(t)$ & Gap in electromagnet and track \\
$F$ & Electromagnetic force \\
$M$ & Total quality of suspension object \\
$h_{p m}$ & Thickness of permanent magnet \\
$g$ & Acceleration of gravity \\
\hline
\end{tabular}

Magnetic density of position is given by

$$
B=\frac{\Phi(z, i)}{A}=\frac{\mu_{0}\left(N i+H_{c} h_{p m}\right)}{2 z(t)+h_{p m} / \mu_{r}} .
$$

Magnetic force $F(t)$ at the moment of $t$ is

$$
F(t)=\frac{B^{2} A}{\mu_{0}}=\mu_{0} A\left[\frac{N i+H_{c} h_{p m}}{2 z(t)+h_{p m} / \mu_{r}}\right]^{2} .
$$

From the above equation, we can see that the relation of hybrid magnetic force, current, and position is nonlinear, but the relation between hybrid magnetic force and magnetic density's square is linear, and the hybrid magnetic force is a single function with regard to magnetic flux density.

The electrical dynamic of hybrid suspension system can be treated as an inductance resistance circuit and formulated as follows:

$$
\begin{aligned}
u(t) & =R i(t)+\frac{d}{d t}[N \Phi(z, i)] \\
& =\frac{R}{N \mu_{p m}}\left[B\left(2 \mu_{r} z+h_{p m}\right)-H_{c} h_{p m} \mu_{p m}\right]+N A \dot{B} .
\end{aligned}
$$


Transform the above equation

$$
\begin{aligned}
\dot{B}=\varphi(x)= & \frac{R}{A \mu_{p m} N^{2}} \cdot\left[H_{c} h_{p m} \mu_{p m}-x_{3} \cdot\left(2 \mu_{r} x_{1}+h_{p m}\right)\right] \\
& +\frac{1}{N A} u .
\end{aligned}
$$

The hybrid magnetic force can be expressed by

$$
M \frac{d^{2} z(t)}{d t^{2}}=M g+f_{d}(t)-F(z, i),
$$

where $f_{d}$ is the external disturbance force.

On all accounts above, the mathematical model of hybrid suspension system in Figure 2 can be described as follows:

$$
\begin{gathered}
B(t)=\frac{\mu_{0}\left(N i(t)+H_{c} h_{p m}\right)}{2 z(t)+h_{p m} / \mu_{r}}, \\
F(t)=\frac{B^{2}(t) A}{\mu_{0}}, \\
M \ddot{z}(t)=-F(t)+M g+f_{d}(t), \\
u(t)=\frac{R}{N \mu_{p m}}\left[B(t) \cdot\left(2 \mu_{r} z(t)+h_{p m}\right)-H_{c} h_{p m} \mu_{p m}\right] \\
+N A \dot{B}(t) .
\end{gathered}
$$

Select state variables

$$
x=\left[\begin{array}{lll}
x_{1} & x_{2} & x_{3}
\end{array}\right]^{T}=\left[\begin{array}{lll}
z & \dot{z} & B
\end{array}\right]^{T} .
$$

The nonlinear hybrid suspension model is given by

$$
\begin{gathered}
\dot{x}_{1}=x_{2}, \\
\dot{x}_{2}=g-\frac{A}{\mu_{0} M} x_{3}^{2}, \\
\dot{x}_{3}=\frac{R}{A \mu_{p m} N^{2}} \cdot\left[H_{c} h_{p m} \mu_{p m}-x_{3} \cdot\left(2 \mu_{r} x_{1}+h_{p m}\right)\right]+\frac{1}{N A} u .
\end{gathered}
$$

So the nonlinear state space equation is obtained:

$$
\begin{gathered}
\dot{x}=f(x)+g(x) u, \\
y=h(x),
\end{gathered}
$$

where

$$
\begin{gathered}
f(x)=\left[\begin{array}{c}
x_{2} \\
g-\frac{A}{\mu_{0} M} x_{3}^{2} \\
\frac{R}{A \mu_{p m} N^{2}} \cdot\left[H_{c} h_{p m} \mu_{p m}-x_{3} \cdot\left(2 \mu_{r} x_{1}+h_{p m}\right)\right]
\end{array}\right], \\
g(x)=\left[\begin{array}{c}
0 \\
0 \\
\frac{1}{N A}
\end{array}\right], \\
h(x)=x_{1}, \quad u=u(t) .
\end{gathered}
$$

$a d_{f}^{2} g(x)$

$=[f,[f, g]]$

$=\frac{\partial\left(a d_{f} g\right)}{\partial x} \cdot f(x)-\frac{\partial f}{\partial x} \cdot\left(a d_{f} g\right)$

$$
=\left[\begin{array}{c}
-\frac{2 x_{3}}{\mu_{0} M N} \\
-\frac{2 R}{A M \mu_{0} \mu_{p m} N^{3}} \cdot\left[H_{c} h_{p m} \mu_{p m}-2 x_{3} \cdot\left(2 \mu_{r} x_{1}+h_{p m}\right)\right] \\
-\frac{2 R \mu_{r}}{\mu_{p m} A^{2} N^{3}} \cdot x_{2}+\frac{R^{2}}{\mu_{p m}^{2} A^{3} N^{5}} \cdot\left(2 \mu_{r} x_{1}+h_{p m}\right)^{2}
\end{array}\right] .
$$

The open loop control block diagram of hybrid suspension system is given by Figure 3 .

Theorem 1 . The nonlinear system (4) and the point $x_{0}$ existing [9]

$$
\begin{gathered}
\dot{x}=f(x)+g(x) u, \\
y=h(x) .
\end{gathered}
$$

The necessary and sufficient condition of the accuracy linearization problem at $x_{0}$ [11] is as follows.

(i) Rank of $H=\left[g, a d_{f} g, \ldots, a d_{f}^{n-1} g\right]$ is $n$, and the term $n$ is also the order of the system.

(ii) The distribution matrix $D_{n-1}=\operatorname{span}\left[g, a d_{f} g, \ldots\right.$, $\left.a d_{f}^{n-1} g\right]$ is involution in neighborhood of $x_{0}$.

2.1. Proof the Necessary and Sufficient Condition of Feedback Linearization. Define the equilibrium point $x_{0}=$ $\left[\begin{array}{lll}z_{0} & \dot{z}_{0} & B_{0}\end{array}\right]^{T}$, the terms $z_{0} \neq 0, \dot{z}_{0}=0, B_{0} \neq 0$. Compute the vector field $g(x), a d_{f} g(x)$, and $a d_{f}^{2} g(x)$ generated by the function $f(x)$ and $g(x)$ :

$$
\begin{gathered}
g(x)=\left[\begin{array}{c}
0 \\
0 \\
\frac{1}{N A}
\end{array}\right], \\
a d_{f} g(x)=L_{f} g(x)-L_{g} f(x) \\
=\left[\begin{array}{c}
0 \\
\frac{2 x_{3}}{\mu_{0} M N} \\
\frac{R}{\mu_{p m} A^{2} N^{3}} \cdot\left(2 \mu_{r} x_{1}+h_{p m}\right)
\end{array}\right],
\end{gathered}
$$




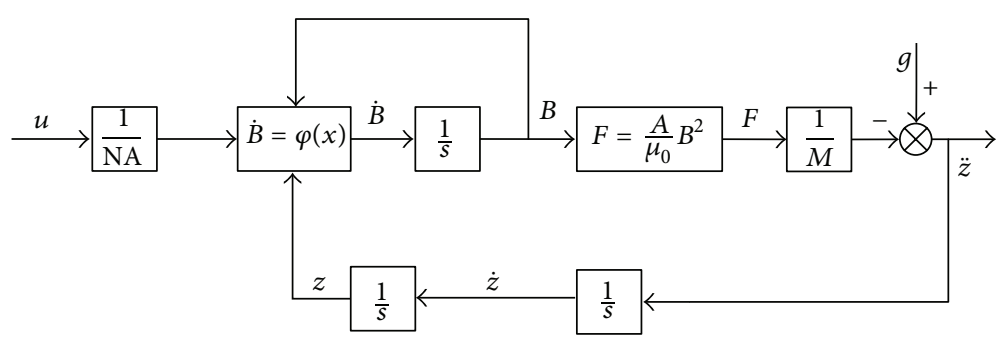

FIGURE 3: Open loop control block diagram of hybrid suspension system.

$$
\begin{aligned}
H & =\left[g(x), a d_{f} g(x), a d_{f}^{2} g(x)\right]_{x_{0}} \\
& =\left[\begin{array}{ccc}
0 & 0 & -\frac{2 R}{A M \mu_{0} \mu_{p m} N^{3}} \cdot\left[H_{c} h_{p m} \mu_{p m}-2 x_{3} \cdot\left(2 \mu_{r} x_{1}+h_{p m}\right)\right] \\
0 & \frac{2 x_{3}}{\mu_{0} M N} & -\frac{2 R \mu_{r}}{\mu_{p m} A^{2} N^{3}} \cdot x_{2}+\frac{R^{2}}{\mu_{p m}^{2} A^{3} N^{5}} \cdot\left(2 \mu_{r} x_{1}+h_{p m}\right)^{2}
\end{array}\right]_{x_{0}} \\
& =\left[\begin{array}{ccc}
\frac{1}{N A} & \frac{R}{\mu_{p m} A^{2} N^{3}} \cdot\left(2 \mu_{r} x_{1}+h_{p m}\right) & -\frac{2 B_{0}}{\mu_{0} M N} \\
0 & \frac{2 B_{0}}{\mu_{0} M N} & -\frac{2 R}{A M \mu_{0} \mu_{p m} N^{3}} \cdot\left[H_{c} h_{p m} \mu_{p m}-2 B_{0} \cdot\left(2 \mu_{r} z_{0}+h_{p m}\right)\right.
\end{array}\right]
\end{aligned}
$$

Because of $\operatorname{rank}(H)=n=3$, the terms $g\left(x_{0}\right), a d_{f} g\left(x_{0}\right)$, $a d_{f}^{2} g\left(x_{0}\right)$ are linearly independent, and also verify that $\operatorname{span}\left\{g(x), a d_{f} g(x), a d_{f}^{2} g(x)\right\}$ is involution distribution in neighborhood of $x_{0}$. So the necessary and sufficient condition of the feedback linearization is satisfied.

2.2. Model of Feedback Linearization. Compute the vector field generated by $f(x), g(x)$, and $h(x)$ :

$$
\begin{aligned}
L_{f}^{3} h(x)= & -\frac{2 R}{A M \mu_{0} \mu_{p m} N^{3}} \\
& \cdot\left[H_{c} h_{p m} \mu_{p m}-2 x_{3} \cdot\left(2 \mu_{r} x_{1}+h_{p m}\right)\right], \\
& L_{g} L_{f}^{2} h(x)=-\frac{2 x_{3}}{\mu_{0} M N} .
\end{aligned}
$$

Design the feedback controller $u=\alpha(x)+\beta(x) v$, the terms

$$
\begin{aligned}
\alpha(x) & =-\frac{L_{f}^{3} h(x)}{L_{g} L_{f}^{2} h(x)} \\
& =-\frac{R}{N \mu_{p m}} \cdot\left[H_{c} h_{p m} \mu_{p m}-x_{3} \cdot\left(2 \mu_{r} x_{1}+h_{p m}\right)\right],
\end{aligned}
$$

$$
\beta(x)=\frac{1}{L_{g} L_{f}^{2} h(x)}=-\frac{\mu_{0} M N}{2 x_{3}} .
$$

Select the transformation of coordinates

$$
\begin{aligned}
z & =\varphi(x) \\
& =\left[\begin{array}{lll}
h(x) & L_{f} h(x) & L_{f}^{2} h(x)
\end{array}\right]=\left[\begin{array}{lll}
z_{1} & z_{2} & z_{3}
\end{array}\right]^{T} .
\end{aligned}
$$

Namely, new state variables are obtained as follow:

$$
\begin{aligned}
z & =\left[\begin{array}{lll}
h(x) & L_{f} h(x) & L_{f}^{2} h(x)
\end{array}\right]^{T} \\
& =\left[\begin{array}{lll}
x_{1} & x_{2} & g-\frac{A}{\mu_{0} M} x_{3}^{2}
\end{array}\right]^{T} .
\end{aligned}
$$




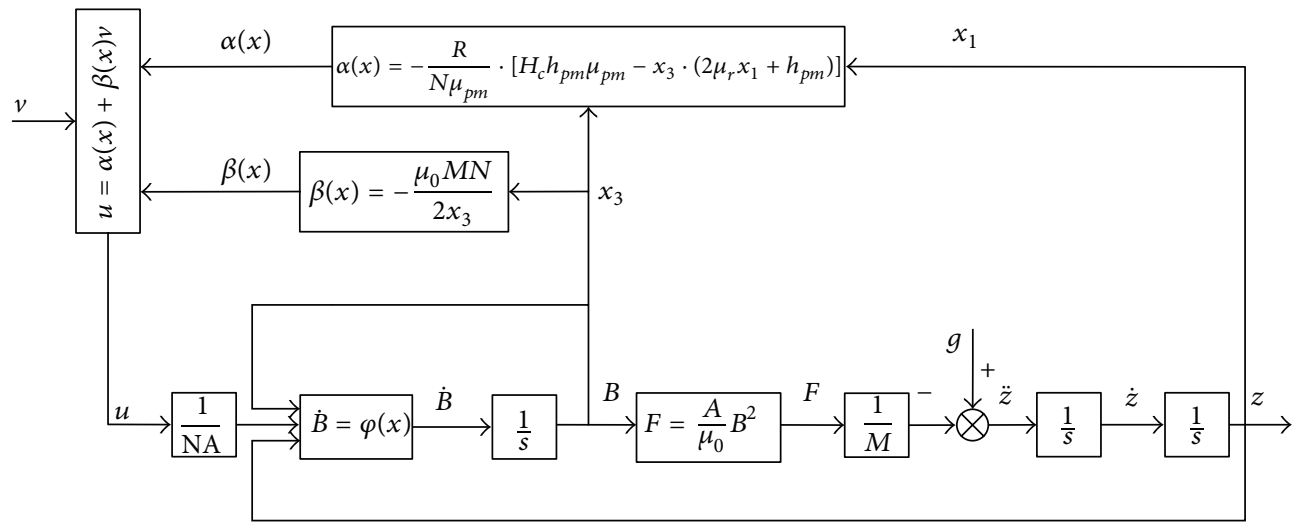

FIGURE 4: Hybrid suspension system block diagram after linearization.

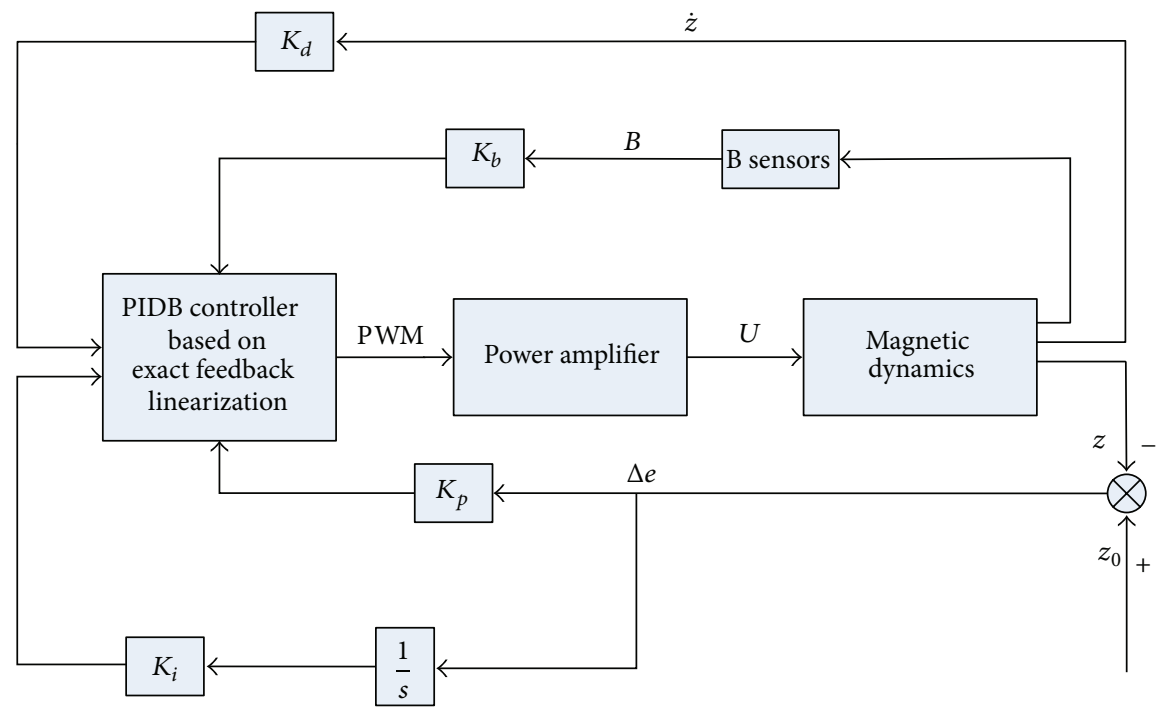

FIGURE 5: PIDB hybrid suspension control system block diagram based on feedback linearization.

In sum, the hybrid suspension system model after linearization:

$$
\begin{gathered}
\dot{z}=\left[\begin{array}{lll}
0 & 1 & 0 \\
0 & 0 & 1 \\
0 & 0 & 0
\end{array}\right] z+\left[\begin{array}{l}
0 \\
0 \\
1
\end{array}\right] v, \\
y=\left[\begin{array}{lll}
1 & 0 & 0
\end{array}\right] z .
\end{gathered}
$$

The maglev system after feedback linearization is a threelevel integral system:

$$
G_{c}=\frac{1}{s^{3}}
$$

The hybrid suspension control block diagram is shown in Figure 4.

\section{Design of Maglev Controller}

The controlled matrix $M_{c}$ of linearizing maglev system is

$$
\operatorname{rank}\left(M_{c}\right)=\left[\begin{array}{lll}
0 & 0 & 1 \\
0 & 1 & 0 \\
1 & 0 & 0
\end{array}\right]=3
$$

Therefore, the hybrid suspension system after linearization is controlled completely, so we can regulate the property of hybrid suspension system by designing PIDB (proportion, integral, differentiation, and magnetic flux density B) suspension controller. Consider

$$
\begin{aligned}
v= & K_{p} \cdot\left(z-z_{0}\right) \\
& +K_{i} \cdot \int\left(z-z_{0}\right) \cdot d t+K_{d} \cdot \dot{z}+K_{b} \cdot B,
\end{aligned}
$$

where $z_{0}$ denotes the given suspension position of hybrid suspension system. 


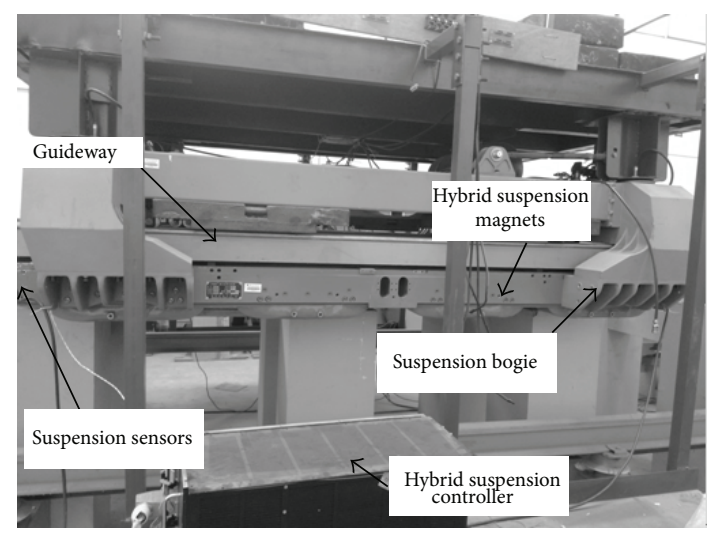

FIGURE 6: CMS04 hybrid suspension experiment platform.

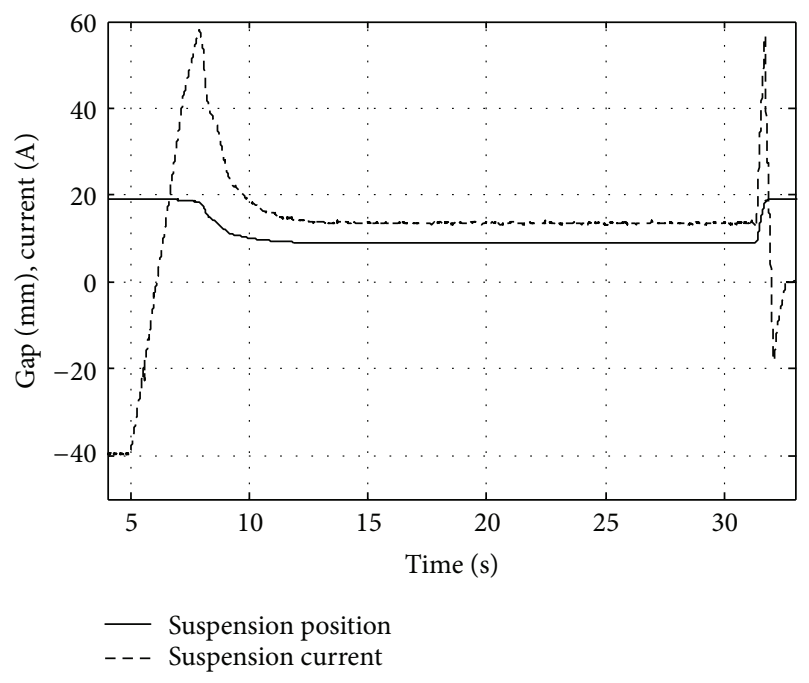

FIGURE 7: Static suspension process of hybrid suspension system.

The closed block diagram of hybrid suspension system is as shown in Figure 5.

\section{Experiments}

The experiment platform is CMS04 hybrid suspension bogie designed by NUDT, showed in Figure 6. Some algorithm experiments have been completed on the standard maglev bogie of the CMS04 maglev vehicle. The first experiment is S1: traditional control algorithm of position-current double cascade control method, and the second is S2: PIDB control algorithm based on state feedback linearization theory designed in this paper. The initial suspension initial position is $25 \mathrm{~mm}$, and the set value is $10 \mathrm{~mm}$. Single standard hybrid suspension bogie's parameters are listed in Table 2.

Experiment 1 (static suspension test). The square quality test on one suspension point of hybrid suspension bogie, setting expected position $9 \mathrm{~mm}$. The experiment applies algorithm S2, and the control parameters are $K_{p}=3200, K_{i}=20, K_{d}=$ 750 , and $K_{b}=260$. The suspension position curve is shown
TABLE 2: Hybrid suspension bogie's parameters of CMS04 maglev vehicle.

\begin{tabular}{lc}
\hline Parameters & Values \\
\hline Total mass $m[\mathrm{~kg}]$ & 653 \\
Turn of magnetic coil $N$ [integer] & 190 \\
Resistance of magnetic coil $R[\Omega]$ & 0.5 \\
Magnetic pole area $A\left[\mathrm{~m}^{2}\right]$ & 0.0235 \\
Equilibrium position $z_{0}[\mathrm{~mm}]$ & 9 \\
Equilibrium magnetic flux density $B_{0}[\mathrm{~T}]$ & 0.6 \\
Equilibrium current $i_{0}[\mathrm{~A}]$ & 0 \\
Magnetic permeability of atmosphere $\mu_{0}$ & $4 \pi \times 10^{-7}$ \\
Magnetic permeability of permanent magnet $\mu_{r}$ & 1.05 \\
Thickness of permanent magnet $h_{p m}[\mathrm{~mm}]$ & 48 \\
Magnetic coercive force of permanent magnet $H_{c}$ & 880 \\
{$\left[\mathrm{kA} \cdot \mathrm{m}^{-1}\right.$ ] } & \\
\hline
\end{tabular}

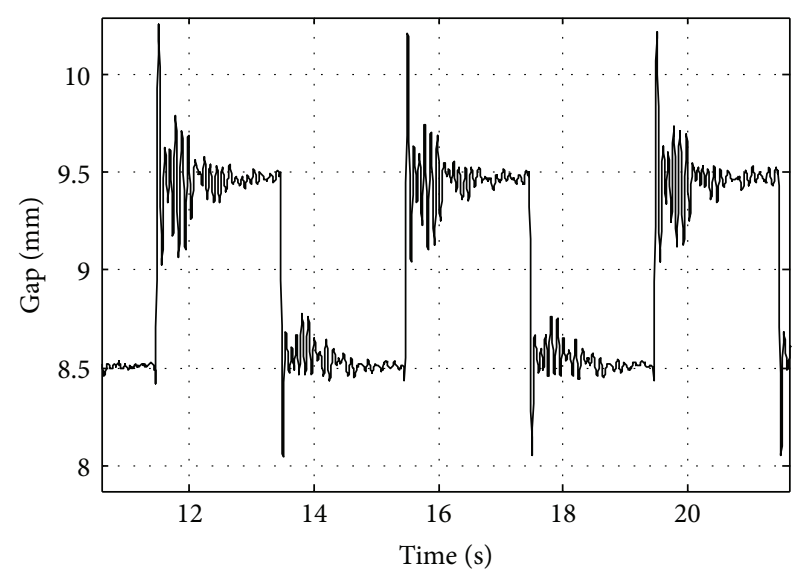

FIGURE 8: Antijamming property test curves of algorithm S1.

in Figure 7. Suspension process is very stable, so it is proved that PIDB control algorithm is scientific and effective.

Experiment 2 (antijamming property test). The square quality test on one suspension point of hybrid suspension bogie, setting the expect position $9 \mathrm{~mm}$, square amplitude $\pm 0.5 \mathrm{~mm}$, respectively, applying two control algorithms S1 and S2. The suspension positions are shown in Figures 8 and 9.

The Experiment 2 results denote that the overshoot of S2 is lower obviously than the one of S1. And the Experiment two result indicates that $\mathrm{S} 2$ has more robustness, better antiinterference property, and better stability than the ones of S1.

\section{Conclusions}

According to hybrid suspension physical model, the hybrid magnetic force is a single function with regard to magnetic flux density B. So we take magnetic flux density signal back to maglev control system and design PIDB hybrid suspension control algorithm based on feedback linearization theory. The PIDB control algorithm has been realized on CMS04 


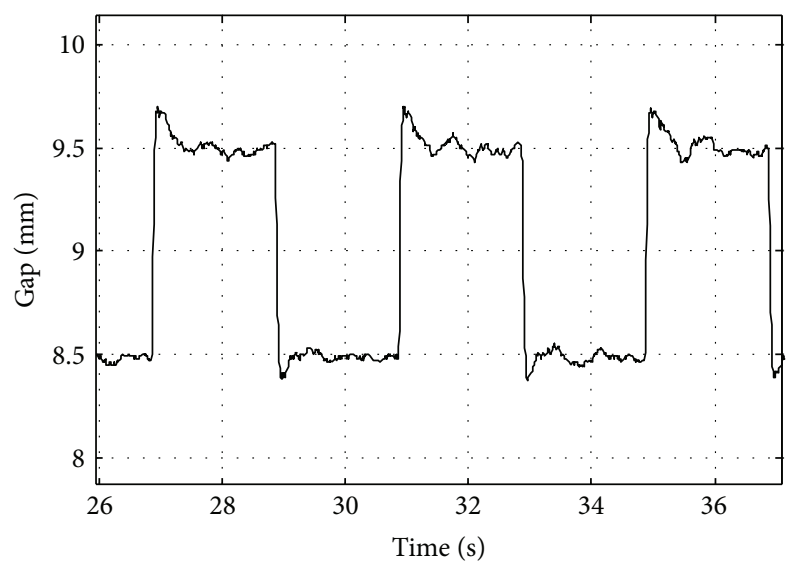

FIGURE 9: Antijamming property test curves of algorithm S2.

hybrid suspension bogie of NUDT with static suspension test and square property test. The experiments illustrate that new method has stronger antijamming quality than traditional position-current double cascade PID control algorithm.

\section{Acknowledgments}

This work was financially supported by National Nature and Science Foundation of China (NNNSFC, nos. 11202230 and 60404003) and the Twelfth Five-year National Science and Technology Support Plan (2012BAC07B01).

\section{References}

[1] R. M. Goodall, "Suspension and guidance control system for a dc attraction Maglev vehicle," in Proceedings of the IEEE Conference, Publication No 142, pp. 100-103, 1976.

[2] W. Zhang, Study and realization on suspension control based on magnetic flux feedback [M.S. Dissertation], National University of Defense Technology, 2009.

[3] R.-J. Wai and J.-D. Lee, "Robust levitation control for linear Maglev rail system using fuzzy neural network," IEEE Transactions on Control Systems Technology, vol. 17, no. 1, pp. 4-14, 2009.

[4] S.-J. Wu, C.-T. Wu, and Y.-C. Chang, "Neural-fuzzy gap control for a current/voltage-controlled 1/4-vehicle MagLev system," IEEE Transactions on Intelligent Transportation Systems, vol. 9, no. 1, pp. 122-135, 2008.

[5] D. Liang, S. Fangzhen, and S. Bo, "Parameters optimization and dynamic characteristic analysis of maglev spindle control system," in Proceedings of the 10th World Congress on Intelligent Control and Automation (WCICA '12), pp. 2403-2406, IEEE Conference Publications, 2012.

[6] Z.-J. Zhang, Y. Liu, J.-J. Cheng, and F. Xiong, "Design and research of a new maglev traffic control system," in Proceedings of the 2nd IEEE International Conference on Advanced Computer Control (ICACC '10), pp. 552-556, March 2010.

[7] R.-J. Wai and J.-D. Lee, "Dynamic control of maglev transportation system via adaptive fuzzy-neural-network," in Proceedings of the International Joint Conference on Neural Networks (IJCNN '07), pp. 569-574, August 2007.
[8] R.-J. Wai and J.-D. Lee, "Adaptive fuzzy-neural-network control for maglev transportation system," IEEE Transactions on Neural Networks, vol. 19, no. 1, pp. 54-70, 2008.

[9] Z. He and W. Wei, "Study on the sliding mode control system for air gap of electrical Maglev based on the feedback linearization," in Proceedings of the 7th World Congress on Intelligent Control and Automation (WCICA'08), pp. 8003-8007, chn, June 2008.

[10] J. Kaloust, C. Ham, J. Siehling, E. Jongekryg, and Q. Han, "Nonlinear robust control design for levitation and propulsion of a maglev system," IEE Proceedings-Control Theory and Applications, vol. 151, no. 4, pp. 460-464, 2004.

[11] C.-K. Chen, C.-J. Lin, and L.-C. Yao, "Input-state linearization of a rotary inverted pendulum," Asian Journal of Control, vol. 6, no. 1, pp. 130-135, 2004. 


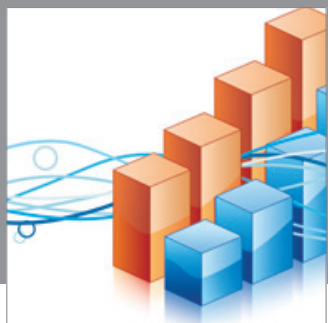

Advances in

Operations Research

mansans

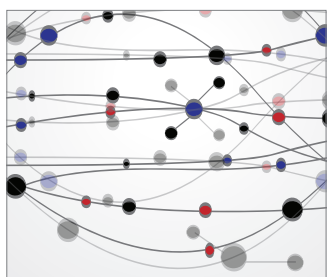

The Scientific World Journal
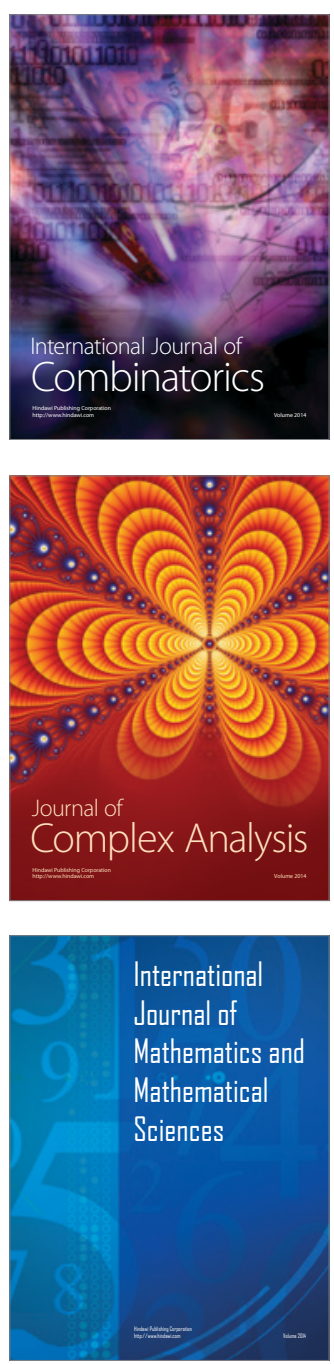
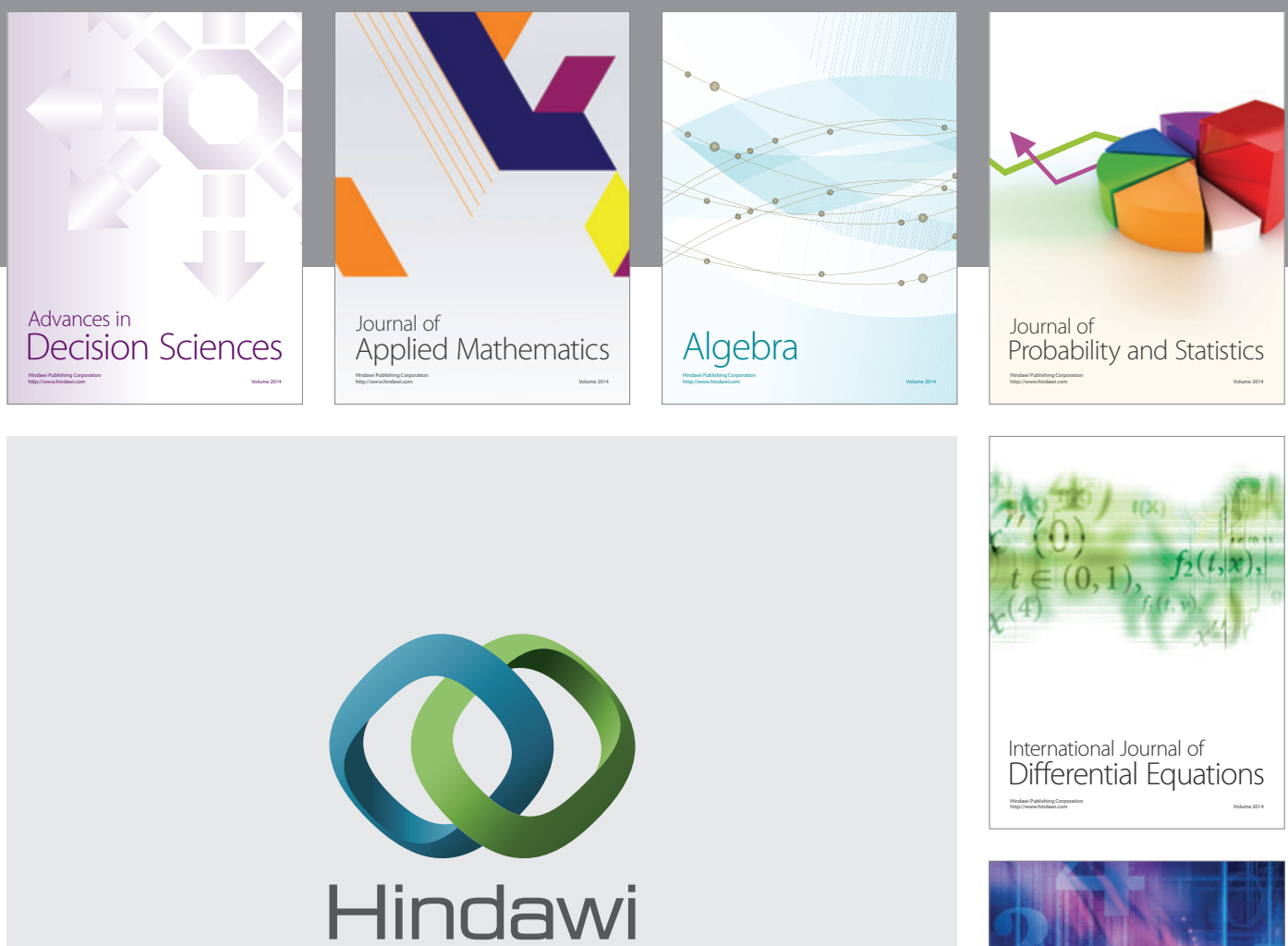

Submit your manuscripts at http://www.hindawi.com
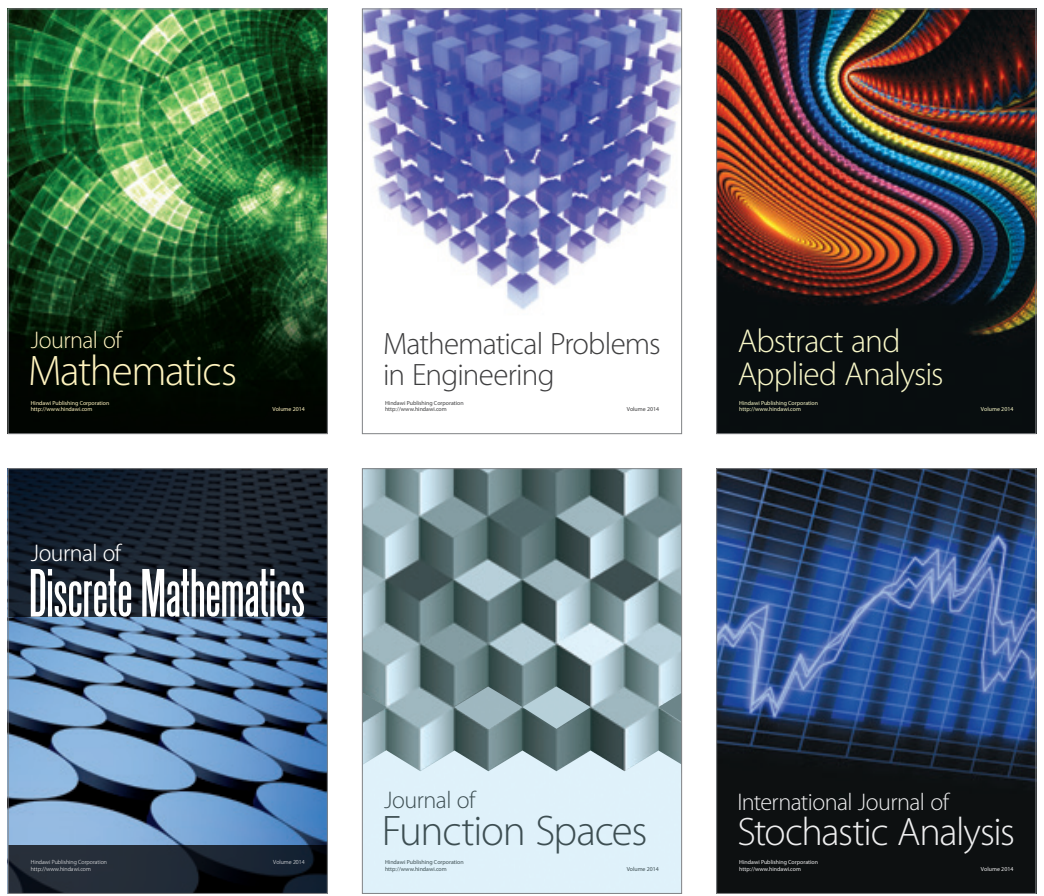

Journal of

Function Spaces

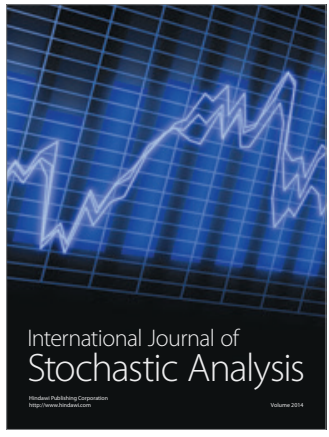

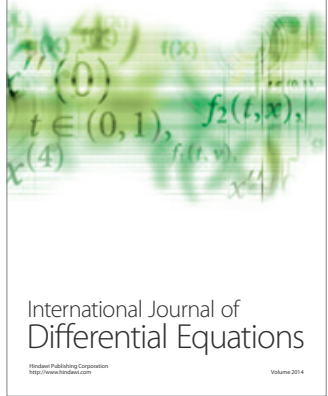
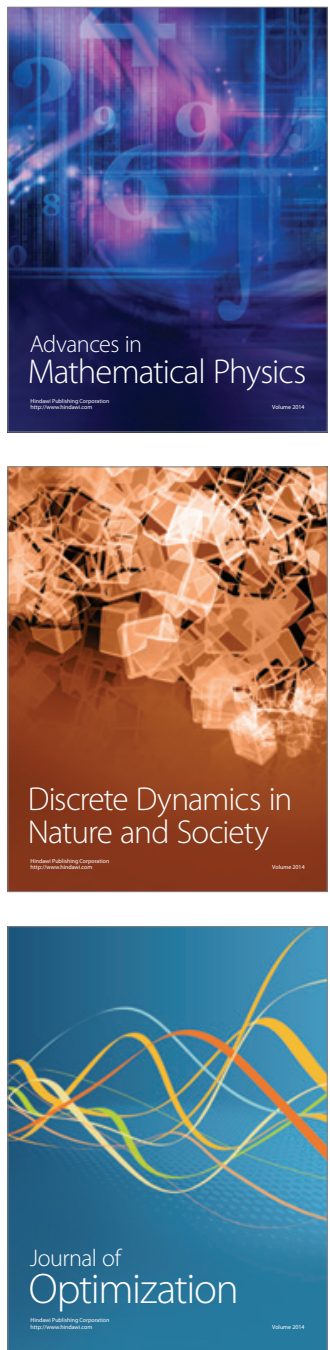